\title{
Automatic Determination of Uric Acid in Urine in a FIA System with a Tubular Amperometric Detector
}

\author{
M. Beatriz Quinaz Garcia, ${ }^{*}$ José L.F.C. Lima, M. Luísa Silva, João P. Sousa ${ }^{a}$ \\ REQUIMTE / Departmento de Química-Física, Faculdade de Farmácia da Universidade do \\ Porto, Rua Aníbal Cunha, 164, 4050-047 Porto, Portugal \\ ${ }^{a}$ Academia Militar, CINAMIL, Rua Gomes Freire, 1150-244 Lisboa, Portugal
}

Received 17 November 2003; accepted in revised form 2 June 2004

\begin{abstract}
This paper describes the use of a FIA system for the automatic determination of uric acid in urine, using a tubular amperometric detector. In this system, glassy carbon electrodes of tubular configuration were activated by successive cyclical scans from - 0.30 to $2.00 \mathrm{~V}$ versus $\mathrm{Ag} / \mathrm{AgCl}$ reference electrode in 0.50 mol. $\mathrm{L}^{-1}$ sulphuric acid solution. Samples were diluted in supporting electrolyte before analysis and no other pre-treatment was employed. Such dilution enabled the matrix effects to be reduced and therefore improving the sensitivity provided by the activation step that allows the uric acid (UA) to be detected at lower concentration levels after dilution. Uric acid determination was performed in a single channel FIA manifold, which provided reproducibility of sample transport to the detector and enabled a sampling rate of 120 samples per hour to be achieved. The tubular configuration of the cell offered robustness to the system since it was rigidly fixed to the manifold, allowing the manifold to be generalised for routine analysis application. The results obtained with the FIA system for UA determination in urine were compared with those from the enzymatic method used in clinical analysis laboratories. No statistical difference between methods was found at the $95 \%$ confidence level. Relative deviations between both methods were within $\pm 4 \%$ and the proposed system showed good repeatability (about 3\%, $\mathrm{n}=10$ ).
\end{abstract}

Keywords: uric acid, amperometric detection, tubular electrodes, FIA.

\footnotetext{
* Corresponding author. E-mail address: bquinaz@ff.up.pt
} 


\section{Introduction}

Uric acid (UA) [7,9-dihydro-1H-purine-2,6,8(3H)-trione], is the principal final product of purine metabolism, being present in biological fluids, especially blood and urine [1]. Its determination enables the detection of alterations associated with the metabolism of purines, for example gout and the Lesch-Nyhan syndrome [1]. Other clinical situations exist in which elevated levels of UA can arise, such as kidney lesion, leukaemia and pneumonia, the UA determination in these cases being used as a complementary diagnostic tool [2].

The clinical importance of UA determination justifies the various methodologies that have been developed, namely colorimetric [3,4], enzymatic [4] and electrochemical [2,5-10]. The colorimetric methods have some limitations, such as the toxic nature of the oxidising agents used, the complex pre-treatment stages and the interference caused by other reducing compounds (such as ascorbic acid), which restrict the application of these methods in routine analysis [4]. The enzymatic methods (with spectrophotometric detection), although very selective, are expensive [4] and are additionally affected by the high absorbance of the matrix itself and by the possibility of enzymatic inhibition caused by purines present in the sample [2].

The electrochemical determination of biological compounds frequently incorporated in complex matrices (as is the case of urine) entails problems related to the selectivity and sensitivity of the method, since the solid electrodes are subjected to the effects of the matrix and can be rapidly contaminated with species which adsorb to their surface (for example purine derivatives) [10] and also suffer interference from species with similar electrochemical behaviour to the species to be measured (in the case of UA determination in urine, reducing species such as ascorbic acid) [2,10]. Furthermore, difficulties in the generalised use of electrochemical methodologies in routine analysis have arisen, namely in clinical analysis laboratories, due to the lack of robustness of the manifolds.

Regarding to surpass these difficulties, an automatic system was developed based on FIA methodology, in which the UA present in the urine is determined 
amperometrically (irreversibly oxidised to yield allantoin), using a tubular detector.

Previous work from our group already stressed that the combination of voltammetric detectors, in their various configurations, with flow systems permits significant advantages to be obtained in routine laboratorial analysis [11]. On the one hand, voltammetric detection enables elevated levels of sensitivity and selectivity to be achieved and, on the other hand, the flow systems permit automation, making viable rapid, reproducible and relatively inexpensive determinations [12-14].

Previous work already demonstrated the efficacy of amperometric detectors of tubular configuration, coupled to FIA systems [11-14], namely in relation to the flexibility of the whole automatic system and the possibility of a rigid fixing to the manifold, conferring it with an elevated degree of robustness.

The utilisation of FIA systems promotes the application of electrochemical procedures in routine clinical analysis since, due to their characteristics, they guarantee the reproducibility of the sample transport to the detector, while easing the conditioning, in the interior of the manifold, of the samples for carrying out the measurements. In addition to this, the system confers simplicity to its own mounting, reduces the consumption of reagents, samples and the production of laboratorial residues and permits elevated sampling rates to be achieved [15]. Furthermore, there is no need for qualified technicians.

The previously referred points justify the development of a FIA system with amperometric detection dedicated to UA determination in urine, where the effects of the complex matrix in which the UA is incorporated and the concentration levels of UA normally found in urine [16] make direct determination, without previous dilution, impossible. With a view to minimising the matrix effect, urine samples were subjected to an elevated dilution in supporting electrolyte solution, before being injected into the system. This dilution requires an elevated degree of sensitivity in the measurement process, to detect reduced concentrations of $\mathrm{UA}$, in the presence of other compounds. In the proposed method, the working electrode was activated electrochemically with the 
objective of increasing the sensitivity of the amperometric determination [10], permitting the surface of the electrode to be increased by promoting the opening of the pores of the glassy carbon structure [17].

\section{Experimental}

\section{Reagents and solutions}

Reagents of p.a. or similar quality were used, without having been subjected to any additional purification. Water purified by the Millipore Milli Q system (conductivity $<0.1 \mu \mathrm{S} . \mathrm{cm}^{-1}$ ) was used throughout.

As carrier solution in the FIA system, a 0.50 mol. $\mathrm{L}^{-1}$ solution of sulphuric acid was used, prepared by dilution of concentrated $\mathrm{H}_{2} \mathrm{SO}_{4}$.

Standard UA solutions used for the attainment of calibration curves in the concentration range of $2 \times 10^{-6}$ to $6 \times 10^{-6} \mathrm{~mol} \mathrm{~L}^{-1}$ were prepared daily, by successive dilutions, from a more concentrated solution $\left(1 \times 10^{-4} \mathrm{~mol} \mathrm{~L}^{-1}\right)$, prepared by rigorous weighting of UA, using a $0.50 \mathrm{~mol} . \mathrm{L}^{-1}$ solution of sulphuric acid as solvent.

Human urine samples were subjected to a 4000-times dilution, in the supporting electrolyte $\left(0.50\right.$ mol. $\mathrm{L}^{-1}$ sulphuric acid solution), in such a way that the concentration of UA present was included in the concentration range of the calibration curve and matrix effects are avoided. The samples did not suffer any additional pre-treatment.

\section{Apparatus}

In the developed FIA manifold (Fig. 1), a Gilson Minipuls 3 peristaltic pump pumped solutions and the intercalation of sample and standards segments was made through an injector-commutator of circular movement [18], with functioning characteristics similar to that with horizontal movement previously referred to in the literature [19]. 


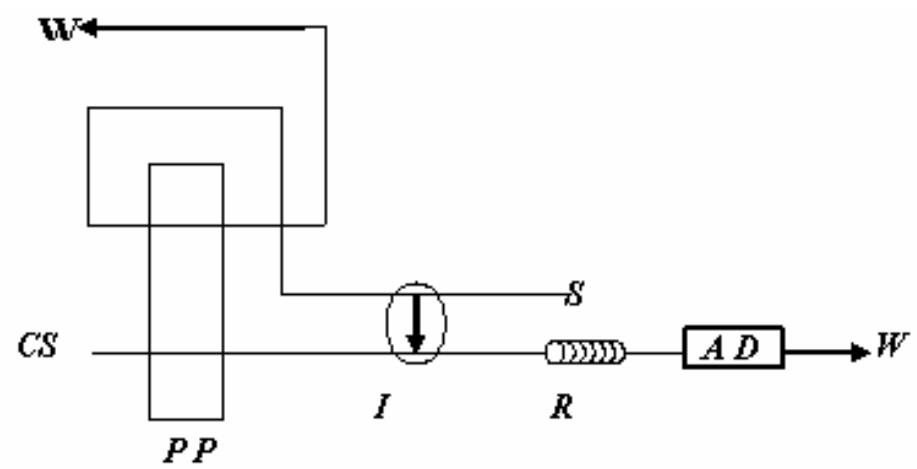

Figure 1. FIA system for uric acid determination in urine. PP peristaltic pump; I injector-commutator $(100 \mu \mathrm{L}) ; \mathrm{R}$ - reactor $(45 \mathrm{~cm}) ; \mathrm{S}$ - sample or standard solution; $\mathrm{AD}$ - amperometric detector; $\mathrm{W}$ - waste; $\mathrm{CS}$ - carrier solution of $0.50 \mathrm{~mol} \mathrm{~L}^{-1}$ sulphuric acid (flow rate of $3.0 \mathrm{~mL} \mathrm{~min}^{-1}$ ).

Connection between the different system components was made through Teflon tubing (Omnifit) of $0.8 \mathrm{~mm}$ internal diameter.

Amperometric measurements were made in an electrochemical system (Eco Chemie, Autolab PGSTAT 10 model) and the data acquisition was made through GPES software, version 4.6.

\section{Tubular amperometric detector}

The tubular amperometric detector (Fig. 2) was constructed in a similar way to that recently referred to in detailed form [12] and was made up of a central Perspex support, where the working and auxiliary electrodes were housed, both of glassy carbon, constructed from a $7 \mathrm{~mm}$ diameter small rod made of this material. The resulting cylinders, of $2 \mathrm{~mm}$ height, were perforated in the centre creating a $1 \mathrm{~mm}$ opening. As reference electrode, a Methrom electrode of $\mathrm{Ag} / \mathrm{AgCl}\left(\mathrm{KCl} 3 \mathrm{~mol} \mathrm{~L}^{-1}\right)$, model 6.0727.000 was used, fixed by a screw thread and superficially touching the solution that flowed in the system. The electrical contact with the working and auxiliary electrodes was established through two metal screws fitted into the perspex support.

At the beginning of each working day, the working electrode $\left(\mathrm{E}_{\mathrm{w}}\right)$ was subjected to a mechanical polishing, using a cotton thread embedded in an aluminium oxide paste (kemet $3 \mu \mathrm{m} \mathrm{3531)}$ for this purpose. 


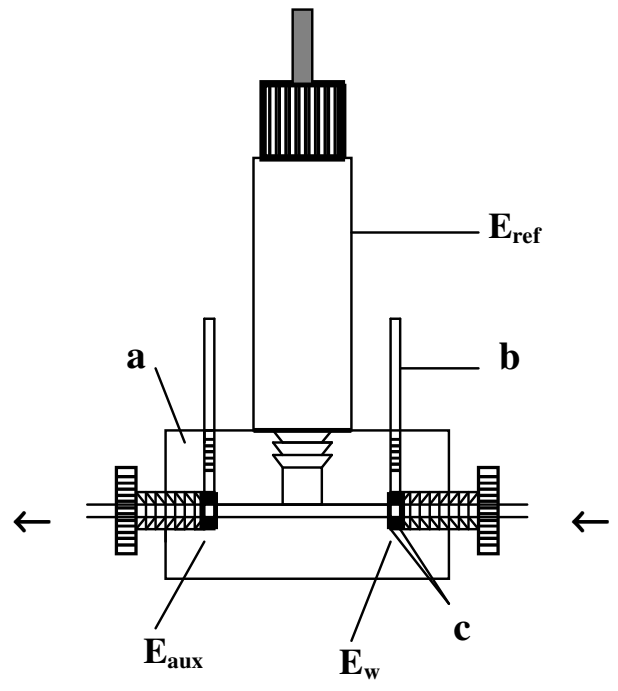

Figure 2. Schematic representation of the electrochemical cell: $\mathrm{E}_{\mathrm{w}}-$ working electrode; $E_{\text {aux }}$ - auxiliary electrode; $E_{\text {ref }}$ - reference electrode; $a$ - perspex support; $b$ - electrical contact; $\mathrm{c}$ - rubber rings; $\rightarrow$ flow direction.

\section{Comparison method}

The UA concentration from the samples analysed by the proposed method was also determined, for comparative purposes, by the enzymatic method used in clinical analysis laboratories. In this method, UA is oxidised by uricase to allantoin and hydrogen peroxide. In the presence of peroxidase, the hydrogen peroxide carries out the oxidative coupling of 3-5-dichloro-2hydroxybenzenesulfonate (DHBS) and 4-aminoantipyrine to form a quinoneimine derivative of red colour. The colour intensity is directly related to the UA concentration and is measured photometrically at $520 \mathrm{~nm}$ [20].

\section{Results and discussion}

\section{Conditioning of the tubular detector working electrode}

As previously referred, at the beginning of each working day, the $\mathrm{E}_{\mathrm{w}}$ was polished and washed with deionised water. Activation was carried out with the detector directly fixed in the FIA system, making the carrier electrolyte solution flow and subjecting the $\mathrm{E}_{\mathrm{w}}$ to a potential cycle between $+2.00 \mathrm{~V}$ and $-0.30 \mathrm{~V}$ [10], with a 
step potential of $0.02 \mathrm{~V}$ and a scan rate of $0.2 \mathrm{~V} . \mathrm{s}^{-1}$. At the end of three cycles, a voltamogram without alterations was obtained.

With the detector incorporated in the flow system, the optimum value of potential to apply to the $E_{\mathrm{w}}$ was also evaluated, sequentially intercalating a $1 \times 10^{-4} \mathrm{~mol} \mathrm{~L}^{-1}$ solution of UA and proceeding to the evaluation of current intensity corresponding to different values of applied potential (from $0.70 \mathrm{~V}$ to $1.60 \mathrm{~V}$ ). In the referred interval, an increase in the amplitude of the analytical signal was observed with the increase in potential, up to a value of $1.20 \mathrm{~V}$, beyond which no further increase in amplitude occurred (Fig. 3). This was the value used in the following assays.

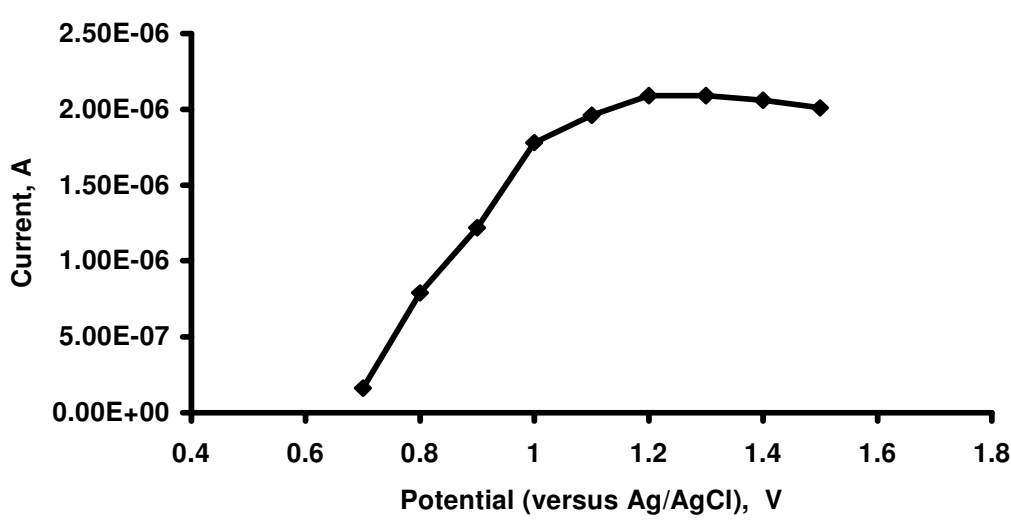

Figure 3. Peak height obtained for a $1 \times 10^{-4} \mathrm{~mol} \mathrm{~L}^{-1} \mathrm{UA}$ solution as a function of the $\mathrm{E}_{\mathrm{w}}$ potential.

Following this, the study of the influence of the activation of the $E_{w}$ on the intensity of the analytical signal was made, intercalating UA solutions of approximately $1 \times 10^{-4} \mathrm{~mol} \mathrm{~L}^{-1}$ concentration in the manifold and evaluating the intensity of the analytical signal in the absence of activation and after the activation of the electrode. It was verified that the analytical signal increased by approximately $60 \%$ after the activation of the electrode, in comparison with the analytical signal in the absence of activation, contributing towards making viable the analysis of significantly diluted urine samples.

Finally, and bearing in mind that an automatic system that permitted the successive analysis of samples was being developed, it was intended to evaluate 
how many determinations could be carried out successively, after an activation stage, without any fall-off in the analytical signal. Repeated determinations were carried out on an approximately $1 \times 10^{-4} \mathrm{~mol} \mathrm{~L}^{-1}$ solution of UA with the respective analytical signal being registered. It was observed that the analytical signal was maintained with the same intensity of current in the first 30 determinations, before beginning to decrease for subsequently injected samples. Therefore, it was only necessary to reactivate the electrode between blocks of 30 determinations, the activation stage taking approximately 1 minute, without being necessary to dismount the system.

\section{Optimisation of the FIA manifold parameters}

In the developed FIA manifold (Fig. 1), standard solutions and samples (S) were injected through an injector-commutator (I) and flowed along a reactor (R) up to the amperometric detector (AD), where the analytical signal was obtained. The injector-commutator movement permitted operation between two positions: in one of the positions, the loop was filled up with the sample or standard solution to analyse and, when rotating to the other position, the loop was intercalated in the principal channel of the system, allowing the supporting electrolyte solution to drag the segment of sample or standard solution up to the detector.

The optimisation of the FIA manifold was carried out with the objective of allowing the rapid determination of UA in urine samples, subjected only to prior dilutions and not requiring any other pre-treatments. Particular attention was given to the optimisation of the injection volume, the length of the reactor that forms the connection between the injection valve and the detector as well as the solutions` flow rate that conditioned the sampling rate and amplitude of the analytical signal.

Regarding the injection volume, values between $20 \mu \mathrm{L}$ and $120 \mu \mathrm{L}$ were tested, using for this purpose loops of length between $4 \mathrm{~cm}$ and $24 \mathrm{~cm}$, composed of teflon tubing of $0.8 \mathrm{~mm}$ internal diameter. Injection volumes greater than $120 \mu \mathrm{L}$ significantly compromised the sampling rate, for a fixed flow rate value, due to the time necessary for the sample segment to fully pass by the detector. Volumes 
between 20 and $50 \mu \mathrm{L}$, although permitting greater sampling rates, gave rise to less reproductive analytical signals, as shown by the relative standard deviation (RSD) values, which were approximately $2 \%(\mathrm{n}=5)$ for a volume of $100 \mu \mathrm{L}$, and never lower than approximately $3 \%(n=5)$ for volumes of 20,40 and $50 \mu \mathrm{L}$, due to the mechanical limitations of the injector-commutator [12]. On this basis, a volume of $100 \mu \mathrm{L}$ was selected for the following tests.

The influence of the reactor length $(\mathrm{R})$, between the injection site (I) and the detector (AD), on the analytical signal was also studied. As it was theoretically predictable, when reduced lengths (between 20 and $30 \mathrm{~cm}$ ) were tested, the analytical signal was more irreproducible (RSD of approximately $2 \%, \mathrm{n}=5$ ) due to an insufficient mixture between the sample and the supporting electrolyte. For greater lengths (between 45 and $200 \mathrm{~cm}$ ), the reproducibility increased (RSD of approximately $1 \%, \mathrm{n}=5$ ) but the analytical signal intensity decreased unnecessarily (approximately $40 \%$ ), due to the increase in dispersion of the sample segment injected and the sampling rate was compromised (for a length of $45 \mathrm{~cm}$ and a flow rate of $3.0 \mathrm{~mL} \cdot \mathrm{min}^{-1}$, the sample segment injected reached the detector in approximately $5 \mathrm{~s}$ and for a length of $2 \mathrm{~m}$, in $20 \mathrm{~s}$ ). A length of $45 \mathrm{~cm}$ was selected, which yielded the best relationship between reproducibility and sampling rate.

Regarding the solutions` flow rate, the influence of its variation on the intensity of the analytical signal was studied, with flow rates between $1.8 \mathrm{~mL} \mathrm{~min}^{-1}$ and $3.4 \mathrm{~mL} \mathrm{~min}^{-1}$ being tested. It was verified that for more reduced flow rates, as expected, the sampling rate was seriously compromised, and for flow rates near $3.4 \mathrm{~mL} \mathrm{~min}^{-1}$, despite the increase in the analytical signal amplitude and the facilitation of greater sampling rates, the reproducibility of the results decreased (the RSD increased for flow rates greater than $3.0 \mathrm{~mL} \mathrm{~min}{ }^{-1}$, reaching the maximum value of approximately $2 \%, \mathrm{n}=5$, for a flow rate of $3.4 \mathrm{~mL} \mathrm{~min}^{-1}$ ). A

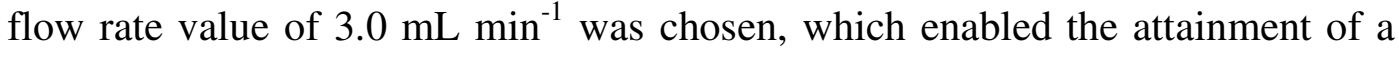
sampling rate of approximately 120 samples per hour and a good reproducibility in the analytical signal (RSD of approximately $1 \%, \mathrm{n}=5$ ). 


\section{Interferences}

One of the greatest problems of in vivo UA determination is the interference caused by some electrochemically active compounds, which can be oxidised under the same conditions of UA, increasing the analytical signal, with the most usual interference being derived from ascorbic acid $[2,10]$. There is also another type of interference caused by species that can be easily adsorbed to the $\mathrm{E}_{\mathrm{w}}$ surface, namely purine derivatives such as caffeine [10].

Considering the results previously published in the literature referring to the UA quantification in urine [10], the method here proposed was developed particularly considering the adsorption problems which, in discrete procedures, require activation of the $E_{w}$ surface after each determination, with the consequent need to systematically dismount the electrochemical cell. As previously mentioned, the dilution to which the samples can be subjected to, permits the decrease of this type of interfering concentration without impeding UA quantification. In addition, the FIA system enables the samples to remain only a short time in contact with the detector (some seconds) and allows a continuous flow of the supporting electrolyte between samples, creating a washing effect of the electrode, this way minimising the adsorption of compounds to the surface of the $\mathrm{E}_{\mathrm{w}}$ and reducing the frequency of activation.

The interference caused by ascorbic acid and caffeine in the UA determination by the proposed FIA methodology was evaluated, by considering an interfering concentration of a compound to be that which gave rise to a $\pm 5 \%$ variation in the analytical signal obtained for a UA solution of given concentration. Therefore, solutions were prepared with a fixed UA concentration and variable concentrations of ascorbic acid and caffeine. It was verified that the presence of ascorbic acid at a concentration equal to that of UA does not cause interference in the determination of the latter; however, for a concentration five times greater, a significant interference was observed. Concerning caffeine, it was verified that, when present at a concentration equal to that of UA, it already interferes in the determination (analytical signal is reduced by approximately 10\%). 
However, these levels of interference do not limited UA determination in urine samples, considering the usual range of urinary concentrations of ascorbic acid (between 10 and $70 \mathrm{mg} \mathrm{L}^{-1}$ ) [20], which is approximately ten times lower than the concentration of UA (250-750 $\mathrm{mg} \mathrm{L}^{-1}$. day $\left.^{-1}\right)$ [16]. Also, ascorbic acid has a reduced stability in urine. Regarding caffeine, its levels in urine are very variable, as they depend on the quantities ingested; however, the quantity of this compound resulting from the ingestion of three to six cups of coffee corresponds to a urinary caffeine concentration of $15 \mu \mathrm{g} \mathrm{mL}^{-1}$ [21], which constitutes a much lower concentration than that at which it can interfere. On this basis, the interference caused by caffeine in UA determination, for the proposed method, is not of any concern.

Table 1. Results obtained in UA determination by the proposed method and the comparison method.

\begin{tabular}{|c|c|c|c|}
\hline \multirow{2}{*}{ Sample } & \multicolumn{3}{|c|}{ UA concentration, mmol L ${ }^{-1}$} \\
\cline { 2 - 4 } & $\begin{array}{c}\text { Proposed } \\
\text { methodology }\end{array}$ & Comparison method & \\
\hline 1 & $1.55 \pm 0.15$ & $1.61 \pm 0.12$ & Relative deviation, $\%$ \\
\hline 2 & $1.49 \pm 0.06$ & $1.50 \pm 0.10$ & -3.73 \\
\hline 3 & $2.30 \pm 0.10$ & $2.35 \pm 0.21$ & -0.67 \\
\hline 4 & $1.99 \pm 0.08$ & $1.95 \pm 0.09$ & +2.13 \\
\hline 5 & $4.08 \pm 0.15$ & $4.05 \pm 0.20$ & +0.74 \\
\hline 6 & $2.15 \pm 0.09$ & $2.08 \pm 0.11$ & +3.37 \\
\hline 7 & $3.75 \pm 0.35$ & $3.70 \pm 0.39$ & +1.35 \\
\hline 8 & $3.82 \pm 0.35$ & $3.75 \pm 0.33$ & -0.82 \\
\hline 9 & $2.41 \pm 0.21$ & $2.43 \pm 0.34$ & 0 \\
\hline 10 & $2.09 \pm 0.17$ & $2.09 \pm 0.06$ & +3.86 \\
\hline 11 & $3.23 \pm 0.11$ & $3.11 \pm 0.57$ & +1.78 \\
\hline 12 & $2.21 \pm 0.04$ & $2.25 \pm 0.13$ & +27 \\
\hline
\end{tabular}

${ }^{\mathrm{a}}$ mean and standard deviation of 3 determinations of each sample. 


\section{UA determination in urine samples}

Considering the predictable values of UA in urine, even in the most serious cases, the system was calibrated by recurring to the triplicate injection of UA standard solutions of concentrations between $2 \times 10^{-6} \mathrm{~mol} \mathrm{~L}^{-1}$ and $6 \times 10^{-6} \mathrm{~mol} \mathrm{~L}^{-1}$. This yielded a linear relationship between the concentrations and the amplitude of the analytical signal obtained. Human urine samples were analysed by the proposed method and the results were compared to the results obtained by the enzymatic methodology, used in clinical analysis laboratories (Table 1).

Correlation between the results obtained by both methods was linear $\left(R^{2}=0.997\right.$, $\mathrm{b}=1.03, \mathrm{a}=0.07$ ) while agreement between the results obtained by both methods was also evaluated by the $\mathrm{t}$ Student test. The calculated $\mathrm{t}$ value $(0.046)$ was lower than the tabulated $t$ value (2.07, two tails) for a confidence level of $95 \%(\mathrm{n}=12)$. To evaluate the reproducibility of the results by the amperometric method, 10 successive injections of a urine sample (with a $3 \mathrm{mmol} \mathrm{L}^{-1}$ concentration) were carried out, yielding a relative standard deviation (RSD \%) of approximately 3\%. The detection limit of the analytical procedure was $6.3 \times$ $10^{-7} \mathrm{~mol} \mathrm{~L}^{-1}$, calculated as being 3 times the standard deviation of background noise [22].

\section{Conclusions}

The developed method permits UA determination in urine, giving results similar to those furnished by current procedures in clinical analysis laboratories, without recourse to sample pre-treatment, in addition to the initial sample dilution. This dilution allows a reduction in the interference degree derived from compounds present in urine and which can be adsorbed to the $\mathrm{E}_{\mathrm{w}}$. On the other hand, the use of a FIA system to transport samples to the detector makes it possible to significantly reduce the contact time between the sample and the electrode. In addition, it minimises the effect of adsorption and enables the continuous flow of 
the supporting electrolyte between samples, thereby creating a washing effect of the electrode and reducing the frequency of activation.

The physical characteristics of the detector permit its easy incorporation in continuous flow manifolds, resulting in a system that is mechanically robust and easily used in the laboratory routine.

The high degree of the detector sensitivity, increased by the activation step, enables analysis of 4000-times diluted samples, the only requirement being the need to repeat the electrode conditioning every 30 determinations, yielding a sampling rate of 120 samples per hour. This suggests the possibility of the proposed method advantageously substitute more expensive methods in terms of time and costs (namely those recurring to enzymes) for UA determination in urine samples.

\section{Acknowledgements}

One of us (M. L. S.) thanks FCT and FSE (III Quadro Comunitário de Apoio) for a Ph.D. grant (BD/10385/02). The authors thank the Clinical Analysis Laboratory of the Faculty of Pharmacy, University of Porto, Portugal, for the urine samples.

\section{References}

1. W.J. Marshall, Clinical Chemistry, $4^{\text {th }}$ edition, Mosby, 2000, 254.

2. R. Bravo, C. Hsueh, A. Jaramillo, A. Brajter-Toth, Analyst 123 (1998) 1625.

3. A.N. Araújo, J.A.M. Catita, J.L.F.C. Lima, Anal. Sci. 14 (1998) 809.

4. M.A.T. Gilmartin, J.P. Hart, B.J. Birch, Analyst 119 (1994) 243.

5. E.T.G. Cavalheiro, K.A. El-Nour, A. Brajter-Toth, J. Braz. Chem. Soc. 11 (2000) 512.

6. $\quad$ H.M. Carapuça, J.E.J. Simão, Port. Electrochim. Acta 11 (1993) 47.

7. R. Bravo, A. Brajter-Toth, Anal. Chem. 44 (1999) 423.

8. X. Cai, K. Kalcher, C. Neuhold, B. Ogorevc, Talanta 41 (1994) 407.

9. R.C. Matos, M.A. Augelli, C.L. Lago, L. Angnes, Anal. Chim. Acta 404 (2000) 151-157.

10. K. Shi, K. Shiu, Electroanalysis 13 (2001) 1319. 
11. R.I.L. Catarino, M.B. Garcia, J.L.F.C. Lima, Port. Electrochim. Acta 17 (1999) 305.

12. R.I.L. Catarino, A.C.L. Conceição, M.B. Garcia, M.L.S. Gonçalves, J.L.F.C. Lima, M.M.C. Santos, J. Pharm. Biom. Anal. 33 (2003) 571.

13. R.I.L. Catarino, M.B. Garcia, R.A.S. Lapa, J.L.F.C. Lima, E. Barrado, J. AOAC Int. 85 (2002) 1253.

14. R.I.L. Catarino, M.B. Garcia, J.L.F.C. Lima, E. Barrado, M. Vega, Electroanalysis 14 (2002) 741.

15. J. Ruzicka, E.H. Hansen, Anal. Chim. Acta 99 (1978) 37.

16. J.D. Bauer, Clinical Laboratory Methods, $9^{\text {th }}$ edition, 1982, 487.

17. M.G. Sullivan, B. Schnyder, M. Bartsch, D. Alliata, C. Barbero, R. Imhof, R. Kotz, J. Electrochem. Soc. 147 (2000) 2636.

18. E.A.G. Zagatto, H. Bergamin Fo, S.M.B. Brienza, M.A.Z. Arruda, A.R.A. Nogueira, J.L.F.C. Lima, Anal. Chim. Acta, 261 (1992) 59.

19. F.J. Krug, H. Bergamin Fo, E.A.G. Zagatto, Anal. Chim. Acta 179 (1986) 103.

20. Todd, Stanford, Davidsohn, Clinical Diagnosis and Management by Laboratory Methods, $17^{\text {th }}$ edition, W.B. Saunders Company, 1984, 138, 1438. National Collegiate Athletic Association, USA.

21. J.N. Miller, Analyst 116 (1991) 3-14. 